\title{
Development and Comparative Study of Effects of Training Algorithms on Performance of Artificial Neural Network Based Analog and Digital Automatic Modulation Recognition
}

\author{
Jide Julius Popoola ${ }^{1}$ and Rex Van Olst ${ }^{2}$ \\ ${ }^{I}$ Centre for Telecommunications Access and Services, School of Electrical and Information Engineering, University of The Witwatersrand, Private \\ Bag 3, Wits 2050, Johannesburg, South Africa \\ ${ }^{2}$ Department of Electrical and Electronics Engineering, School of Engineering and Technology, Federal University of Technology, P.M.B. \\ 704, Akure, Ondo State, Nigeria
}

Received 8 December 2014; Accepted 2 November 2015

\begin{abstract}
This paper proposes two new classifiers that automatically recognise twelve combined analog and digital modulated signals without any a priori knowledge of the modulation schemes and the modulation parameters. The classifiers are developed using pattern recognition approach. Feature keys extracted from the instantaneous amplitude, instantaneous phase and the spectrum symmetry of the simulated signals are used as inputs to the artificial neural network employed in developing the classifiers. The two developed classifiers are trained using scaled conjugate gradient (SCG) and conjugate gradient (CONJGRAD) training algorithms. Sample results of the two classifiers show good success recognition performance with an average overall recognition rate above $99.50 \%$ at signal-to-noise ratio (SNR) value from $0 \mathrm{~dB}$ and above with the two training algorithms employed and an average overall recognition rate slightly above $99.00 \%$ and $96.40 \%$ respectively at $-5 \mathrm{~dB}$ SNR value for SCG and CONJGRAD training algorithms. The comparative performance evaluation of the two developed classifiers using the two training algorithms shows that the two training algorithms have different effects on both the response rate and efficiency of the two developed artificial neural networks classifiers. In addition, the result of the performance evaluation carried out on the overall success recognition rates between the two developed classifiers in this study using pattern recognition approach with the two training algorithms and one reported classifier in surveyed literature using decision-theoretic approach shows that the classifiers developed in this study perform favourably with regard to accuracy and performance probability as compared to classifier presented in previous study.
\end{abstract}

Keywords: Automatic modulation recognition (AMR), modulation recognition families, AMR development approaches, artificial neural networks classification.

\section{Introduction}

In recent years, the means of carrying out nearly all operations has dramatically shifted from manually operated system to an automatic system. This is as a result of some advantages that an automatic operated system exhibits over its manual counterpart. For instance, recognition of modulation schemes in wireless communication was basically manually operated. However, in the recent years the manual approach has been replaced with automatic systems. This is because some observed disadvantages in manual modulation recognition systems such as slow response rate in a hostile signal environment and its success which depends largely on operator's experience [1] has been overcome in automatic modulation recognition with fast response rate and without human involvement [2]. The tool recently developed for monitoring or recognizing the wireless modulated signals in an automatic way is called automatic modulation recognition.

Automatic modulation recognition (AMR), also known

\footnotetext{
* E-mail address: jidejulius2001@gmail.com

ISSN: 1791-2377 @ 2015 Kavala Institute of Technology. All rights reserved.
}

as automatic modulation classification (AMC) as reported in [3] is defined as a tool used in recognizing the type of modulation scheme used to generate a received modulated signal without any a priori knowledge of the modulated signal itself. Similarly, it was also defined in [4] as an intermediate operation between the signal detection and data demodulation, which plays an important role in both military and civilian applications such as electronic warfare, surveillance, threat analysis, signal confirmation, signal interference identification and spectrum management.

Basically, performing AMR is a challenge owing to the lack of a priori knowledge about the modulated signal. This lack of a priori knowledge about the modulated signal is essence because algorithms for AMR are expected to be blind in nature. Hence, meeting this expectation has made development of AMR algorithm an interesting research area in the recent years with various algorithms developed for different modulation schemes.

Since research on AMR commenced about three decades ago, three distinct families of AMR have been developed. The first family of AMR classifier developed are those that were designed to recognize only analog modulated signals [2,5-9]. The classifier in the second family are those that are developed to recognise only digital modulated signals [10- 
15] while the third family of the AMR classifier are those that are designed to recognise or classify some joint analog and digital modulated signals [16-18]. These few selected algorithms: [2,5-9], [10-15] and [16-18] for first, second and third families of AMR are briefly reviewed in Section 2.

In general, irrespective of the family that an AMR belongs to, surveyed literature revealed that two methods or approaches are normally employed in AMR algorithm development. The two approaches normally employed are the decision-theoretic (DT) approach and the pattern recognition (PR) approach [4, 14]. In DT approaches, probabilistic and hypothesis testing arguments are used to formulate the recognition problem. In using this approach, it is assumed that each possible modulation scheme happens with the same probability. Hence, the maximum likelihood (ML) criterion is being employed. The application of the ML criterion to either the received signal directly or to a certain transform of the received signal results in a likelihood ratio or set of likelihood function. In this approach, the recognition decision is decided by comparing the likelihood functions or by comparing the likelihood ratio with a threshold. The solution offered by the DT based algorithm is optimal since the approach minimizes the probability of false recognition or classification [19]. However, the optimal solution usually suffers from heavy computational complexity [19]. Other two drawbacks of this approach are the difficulties involved in forming the right hypothesis for the approach and how to carefully set the correct threshold values for each feature keys employed. As a result of these drawbacks, DT based AMR naturally gives rise to suboptimal classifiers.

On the other hand, PR approaches do not require any careful treatment. According to [14], the only challenge with this approach is how to choose the right set of feature extraction keys since the approach employs one or several feature keys from the received signal to make decisions. In overcoming this challenge, PR modulation recognition module is divided into two subsystems, namely the feature extraction subsystem and the pattern recognizer subsystem $[17,19,20]$. In the first subsystem of this approach, feature extraction keys are extracted from the radio signal. Some of the commonly adopted feature extraction keys are higherorder statistics (HOS), including moments, cumulants, and cyclic cumulants (CC) of the signal $[4,19,20]$, fuzzy logic [21,22], a constellation shape recovery method [23] and usage of information contained in an incoming signal [2,17, 24-26]. The second subsystem of the PR approach is a pattern recognizer, which processes those feature keys and determines the modulation type of the received signal according to a pre-designed decision rule. Multi-Layer Perceptron Neural Network is one of the classifiers that are used in modulation recognition systems. It has been shown that this type of classifier outperforms other classifiers, such as the K-nearest neighbourhood algorithm [17].

In contrast to the DT approaches, the PR approaches are non-optimal, but they are more robust and simple to implement. Most often, according to [4], if PR approaches are carefully designed, they can achieve nearly optimal performance. Therefore, the study presented in this paper adopted a PR approach with application of ANN as its model classifier. The choice of ANN as the model classifier for this study was not based only on its advantage over other classifiers as mentioned above but to also verify the hypothesis made by [27] that training algorithms has significant impact on the performance of ANN and the fact that the effects of the training algorithms depend on the targeted application. These authors verified their hypothesis by considering the effect of training algorithms on a multilayer feed forward ANN classifier for image coding. In order to scientifically investigate their observation, the focus of the study reported in this paper, which is an expanded version of our recently presented paper at IEEE AFRICON 2013 Conference, is centred on an investigation of the effect of training algorithms on performance of a joint analog and digital AMR recognizer or classifier using ANN. The study presented in this paper on modulation schemes classification is considered as an ideal study to verify the hypothesis because, classification has been observed as one of the most active research and application areas of ANN [28].

The paper, therefore, presents a comparative study on effects of two different training algorithms on performance of multilayer feed forward ANN with back propagation algorithm in joint analog and digital modulations classification. The remainder of the paper is organized as follows. Section 2 provides brief review of the three families of AMR. Section 3 provides detailed information on the procedures involved in developing the AMR for this study. The simulation results and comparative performance evaluation of the developed AMR classifiers for this study based on the two training algorithms employed are presented and discussed in Section 4. Finally, Section 5 concludes the paper.

\section{Automatic Modulation Recognition Review}

This section is concerned with the review of research papers since 1984 in the field of modulation recognition. The section is divided into three sub-sections according to the three AMR families. The first sub-section is concerned with brief review of analog automatic modulation recognitions (AMRs). The second and third sub-sections are concerned with brief review of both digital AMRs and joint analog and digital AMRs respectively. Analysis of these three classes of AMR algorithms shows that different features are employed in their development.

\subsection{Review of selected analog modulated signals recognition algorithms}

In 1985, Chen et al [5], developed a modulation recognizer for analog modulation schemes based on analytic expressions of the ratio of the variance of the instantaneous amplitude (IA) to the square of the mean value of IA for the amplitude modulation (AM), double sideband (DSB) modulation, and frequency modulation (FM). In Fabrizi et al [6], the authors developed an AMR recognizer that employed the features based on the variations of both the instantaneous amplitude and instantaneous frequency. The feature keys used by these authors are the ratio of the envelope peak to its mean and the mean of the absolute value of the instantaneous frequency. With the aid of these feature keys, the AMR classifier by these authors was used to discriminate between carrier wave $(\mathrm{CW}), \mathrm{AM}, \mathrm{FM}$ and single sideband (SSB) modulation.

In Al-Jalili [7], the centralized instantaneous frequency (CIF) was studied, which is the ratio of the number of the positive spikes to that of the negative spikes was used to discriminate between the upper sideband (USB) and lower sideband (LSB) modulation schemes. Azzouz and Nandi [8] also proposed an analog AMR recognizer that employed feature keys extracted from the instantaneous amplitude and instantaneous phase as well as the radio frequency signal 
spectrum. The authors used a decision theoretic approach with this aid of the extracted feature keys to discriminate between AM, DSB, LSB, USB, vestigial sideband (VSB) modulation, FM and combined modulated signals. In 2005 Sengur and Guldemir [9] developed an analog AMR that employed instantaneous amplitude and instantaneous phase as well as the radio frequency signal spectrum to discriminate between AM, FM and CW modulations. In 2007 again, Guldemir and Sengur [2] developed another AMR that also employed instantaneous amplitude and instantaneous phase as well as the radio frequency signal spectrum to discriminate AM, FM, DSB, USB, LSB and CW modulations.

\subsection{Review of selected digital modulated signals recognition algorithms}

Similarly, the development of digitally modulated AMR algorithms surveyed revealed that Liedtke [10] was the first author to publish modulation recognition of digitally modulated signals. The author's digital AMR utilises the universal demodulator technique that employed the amplitude histogram, the frequency histogram, the phase difference histogram, the amplitude variance and the frequency variance as the feature key. The recogniser introduced by this author was used to recognise or classify two symbol amplitude shift keying (2ASK), two symbol frequency shift keying (2FSK), two symbol phase shift keying (2PSK), four symbol phase shift keying (4PSK), eight symbol phase shift keying (8PSK) and CW modulations. In DeSimio and Prescott [11], an adaptive technique was employed for classifying 2ASK, 2FSK, 2PSK and 4PSK using the feature keys extracted from the signal envelope, the signal spectra, the signal squared and the fourth power of the signal. Azzouz and Nandi [12] in addition proposed a digitally modulation recognizer that discriminated between 2ASK, 4ASK, 2FSK, 4FSK, 2PSK and 4PSK. The feature keys used for developing this modulation classifier were extracted from the instantaneous amplitude, instantaneous phase and instantaneous frequency of the signal under consideration.

In addition, in Taira [13], the histogram distribution of instantaneous amplitude at symbol points was used for the automatic classification of digitally quadrature amplitude modulation (QAM) signals including 64-state QAM and 256-state QAM. The author obtained good classification results by computer simulations at SNR greater or equal to $10 \mathrm{~dB}$. In Wong and Nandi [14], an ANN based digital classifier was developed to recognise ten digital modulation schemes, namely 2ASK, BPSK, 2FSK, 16QAM, V29, V32, 4ASK, QPSK, 4FSK and 64QAM. In developing their classifier, a total of seven feature extraction keys were used. Five of these seven feature extracted keys were derived from the instantaneous amplitude, instantaneous phase and instantaneous frequency of the signals. These five feature keys were earlier used in [12] for discriminating between digital signals that have hidden information in a single domain. The modulated signals these five keys were used to discriminate were 2ASK, BPSK, 2FSK, V29, V32, 4ASK, QPSK, and 4FSK while the two additional feature set keys a spectral feature set and a new feature set based on higherorder cumulants were used to discriminate between 16QAM and 64QAM that have information content hidden either in the signal instantaneous amplitude, instantaneous phase or instantaneous frequency.

Furthermore in 2011, Kubankova and Kubanek [15] successfully developed a digital modulation classifier that employed the maximum value of the spectral power density of the normalized-centered instantaneous amplitude of the received signal as the distinguishing feature keys to discriminate between ASK, 2FSK, 4FSK, MSK, BPSK, QPSK and 16QAM digital modulations used in modern communication technologies. The developed recognizer was tested with simulated and measured signals corrupted by white Gaussian noise. The results show that for higher SNR of $15 \mathrm{~dB}$, the measured signals are correctly recognized only with slightly lower probability than the simulated ones.

\subsection{Review of selected joint and digital modulated signals recognition algorithms}

In 1985, Jondral [16] proposed a modulation recognizer that utilized the pattern recognition method for the noise signal and two types of analog modulated signals namely AM and SSB as well as for four types of digitally modulated signals namely 2ASK, 2FSK, 4FSK and 2PSK. In developing the joint analog-digital recognizer, the feature keys employed were extracted from instantaneous amplitude, phase and frequency. Similarly, in 1998, Nandi and Azzouz [17] introduced two separate algorithms for joint analog and digital modulations recognition. The first algorithm developed by these authors utilized the decision-theoretic approach while the second algorithm developed utilized the ANN as a new approach. The joint analog-digital modulations recognition were developed to recognize joint AM, DSB, VSB, LSB, USB, FM and combined modulated signals analog modulations and 2ASK, 4ASK, 2FSK, 4FSK, 2PSK and 4PSK digital modulations. The feature keys used in the two proposed algorithms were extracted from the instantaneous amplitude, instantaneous phase and instantaneous frequency of the signals except the signal spectrum symmetry key which was extracted from the radio frequency (RF) signal spectrum. Evaluation of the two algorithms revealed that the ANN algorithm outperformed the decision-theoretic method with overall success rate over $96 \%$ against over $94 \%$ at SNR values of $15 \mathrm{~dB}$. Also, in 2005, Dobre et al [18] developed a different joint analog and digital AMR algorithm based on a set of five feature keys obtained from the baseband of the received signal. The algorithm was used to discriminate the following pool of analog and digital modulations: AM, DSB, USB, LSB, BPSK, QPSK, 8PSK, 16QAM and 32QAM

In this paper we propose a new combined analog and digital AMR based on a set of eight feature keys. Seven of these feature keys were derived from the instantaneous amplitude and the instantaneous phase of the simulated signals while the eighth feature key was derived from the signal spectrum symmetry of the RF signal spectrum of the simulated signal. The proposed algorithm is applied to discriminate the following combined analog and digital modulations: 2ASK, 4ASK, 2FSK, BPSK, QPSK, AM, DSB, SSB, FM, orthogonal frequency division multiplexing (OFDM), 16QAM and 64QAM. In comparison with the three earlier work [16-18] on joint or combined analog and digital modulations recognition, the algorithm proposed in this study is the only one that includes 64 state QAM and OFDM. The detailed information on the procedure involved in developing the proposed algorithm for this study is presented in next section.

\section{The AMR Developmental Stages}

In this study, the PR approach or methodology for developing AMR was employed. Hence, the activities under 
the two subsystems in PR approach for AMR development were strictly observed. Detailed information on activities involve in the two subsystems are presented in the following subsystems.

\subsection{AMR pattern recognition approach first subsystem}

This subsystem is known as the feature keys extraction subsystem. It deals with the extraction of feature keys that will be used as input to the classifier in the second subsystem. This subsystem is in essence an AMR development because its success has a significant effect on the accuracy of the desired AMR. Hence, in developing the AMR for this study, a total of eight feature keys were used. Seven of the keys were extracted from information hidden in the instantaneous amplitude and the instantaneous phase of the combined simulated modulated signals. The last feature key was derived from the information hidden in the signal spectrum symmetry of the RF signal spectrum of the simulated signal. These feature extraction keys had been used in some earlier studies in the literature. However, there are no such studies that used all the eight keys together as used in this study.

The first feature extraction key used is $\gamma_{\max }$, which represents the maximum value of the power spectral density of the normalized instantaneous amplitude of the signal, or, simply put, as the squared Fourier transform of the normalized signal amplitude. It is defined in $[25,26]$ as;

$\gamma_{\max }=\max \frac{\left|\operatorname{DFT}\left(a_{c n}(i)\right)\right|^{2}}{N}$

where $N$ is the number of samples per segment and $a_{c n}(i)$ is the value of the normalized-centered instantaneous amplitude of the signal at time instants $t=i / f_{s}(i=1,2, \cdots, N), f_{s}$ is the sampling frequency $(\mathrm{Hz})$ and $a_{c n}(i)$ is defined as:

$a_{c n}(i)=a_{n}(i)-1$

and;

$a_{n}(i)=\frac{a(i)}{m_{a}}$

where $m_{a}$ is the average value of the instantaneous amplitude evaluated over one segment. It is defined as:

$m_{a}=\frac{1}{N} \sum_{i=1}^{N} a(i)$

The second feature extraction key used is, $\sigma_{d p}$, which is the standard deviation of the direct instantaneous phase of the of the simulated signal. It extracts information from the instantaneous phase of the simulated signal. $\sigma_{d p}$ is defined in $[25,26]$ as;

$$
\sigma_{d p}=\sqrt{\frac{1}{C}\left(\sum_{a_{n}(i)>a_{t}} \varphi_{N L}^{2}(i)\right)-\left(\frac{1}{C} \sum_{a_{n}(i)>a_{t}} \varphi_{N L}(i)\right)^{2}}
$$

where $\varphi_{N L}(i)$ is the value of the non-linear component of the instantaneous phase at time instants $t, C$ is the number of the samples in $\varphi_{N L}(i)$, and $a_{t}$ is the threshold.

The third feature extraction key used is for measuring the spectrum symmetry around the carrier frequency. This feature extraction key is based on the spectral powers for the lower and upper sidebands of the simulated signal. The key is defined in $[2,25,26]$ as;

$P=\frac{P_{L}-P_{U}}{P_{L}+P_{U}}$

where

$$
P_{L}=\sum_{i=1}^{f_{c n}}\left|X_{c}(i)\right|^{2} \text { and } P_{U}=\sum_{i=1}^{f_{c n}}\left|X_{c}\left(i+f_{c n}+1\right)\right|^{2}
$$

where $X_{C}(i)$ is the Fourier transform of the intercepted signal, $\left(f_{c n}+1\right)$ is the sample number corresponding to the carrier frequency $f_{c}$ and $f_{c n}$ is defined as

$f_{c n}=\frac{f_{c} N}{f_{s}}-1$

The fourth feature extraction key employed for the development of the AMR recognizer for this study is $v_{20}$, which is the combined or mixed order moments. Based on the Joint Power Estimation and Modulation Classification algorithm, $v_{20}$ is defined mathematically in [29] as;

$v_{20}=\frac{M_{4,2}(y)}{M_{2,1}^{2}(y)}=\frac{E\left(|y(n)|^{4}\right)}{E\left(|y(n)|^{2}\right)}=\frac{m_{20}(S / N)^{2}+4(S / N)+2}{(S / N)^{2}+2(S / N)+1}$

where

$m_{20}=\frac{M_{4,2}(u)}{M_{2,1}^{2}(u)}=2 k_{20}$

The theoretical values of $k_{20}$ for 16-QAM, 64-QAM and OFDM employed in this study according to [30] are 1.312, 1.378 and 2.0 respectively.

The fifth feature extraction key employed in this study is signal power key denoted as $\beta$. This key was used to discriminate between a signal with complex and real signals components. Mathematically, it is defined in [18] as;

$\beta=\frac{\int_{-\infty}^{\infty} r_{Q}^{2}(t) d t}{\int_{-\infty}^{\infty} r_{I}^{2}(t) d t}$ 
where $r_{Q}(t)$ and $r_{I}(t)$ are the quadrature components, while indexes $I$ and $Q$ stand for in-phase and quadrature component respectively.

The sixth feature extraction key used is the mean value of the amplitude designated as $X$. It is defined mathematically by [31] as:

$$
X=\frac{1}{N} \sum_{n=1}^{N} A_{n}
$$

where $A_{n}$ is the instantaneous amplitude. This key was used to discriminate between 16-QAM as a subset and 64-QAM as the other subset.

The seventh feature key used in this study is $\sigma_{a p}$, which is the standard deviation of the absolute value of the nonlinear component of the instantaneous phase. It is defined in [26] as:

$\sigma_{a p}=\sqrt{\frac{1}{C}\left(\sum_{a_{n}(i)>a_{t}} \varphi_{N L}^{2}(i)\right)-\left(\frac{1}{C} \sum_{a_{n}(i)>a_{t}} \mid \varphi_{N L}(i)\right)^{2}}$

where $\varphi_{N L}(i)$ is the value of the non-linear component of the instantaneous phase at time instants $t=i / f_{s}, C$ is the number of the samples in $\varphi_{N L}(i)$, and $a_{t}$ is the threshold.

The eighth feature extraction key used in developing the AMR for this study is $\sigma_{a a}$. It is the standard deviation of the absolute value of the normalized instantaneous amplitude of the simulated signal. It is defined as in [26]:

$$
\sigma_{a a}=\sqrt{\frac{1}{N}\left(\sum_{i=1}^{N} a_{c n}^{2}(i)\right)-\left(\frac{1}{N} \sum_{i=1}^{N}\left|a_{c n}(i)\right|\right)^{2}}
$$

This feature keys extraction activity was carried out in MATLAB environment. The graphical representations of the eight keys from the MATLAB environment are shown in Fig. 1(a-h).

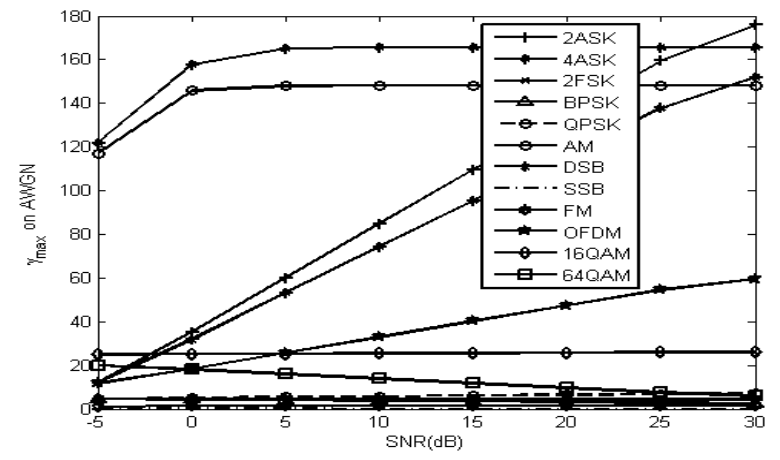

(a)

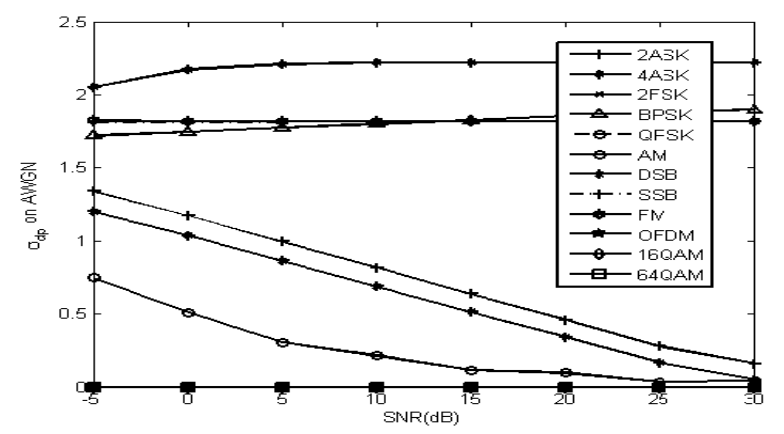

(b)

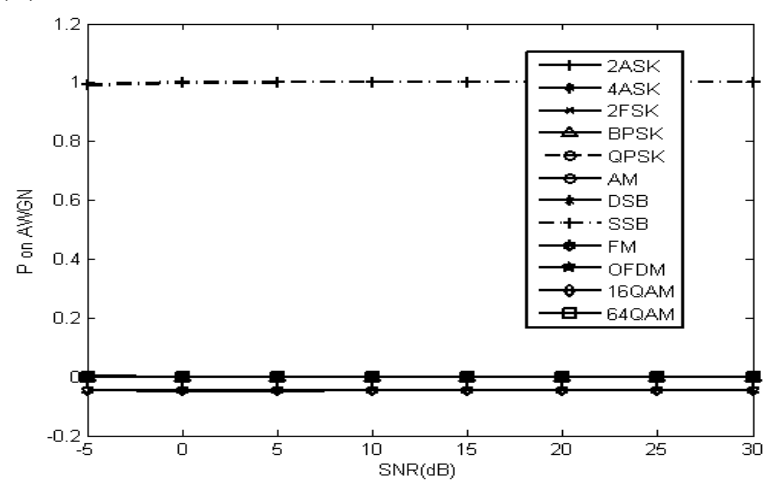

(c)

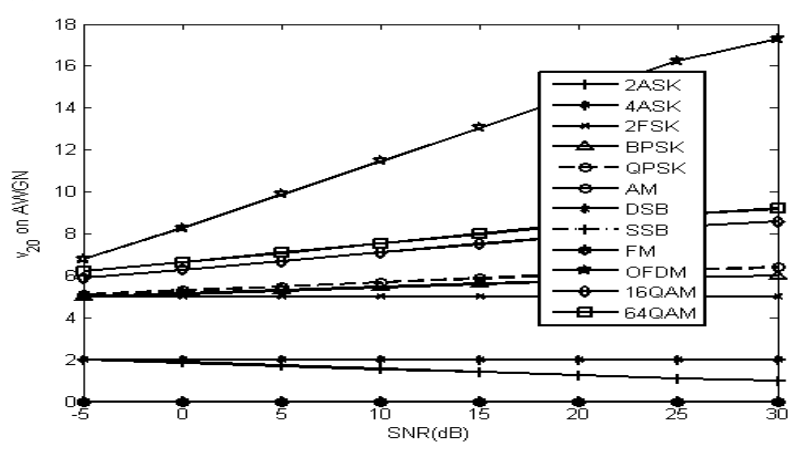

(d)

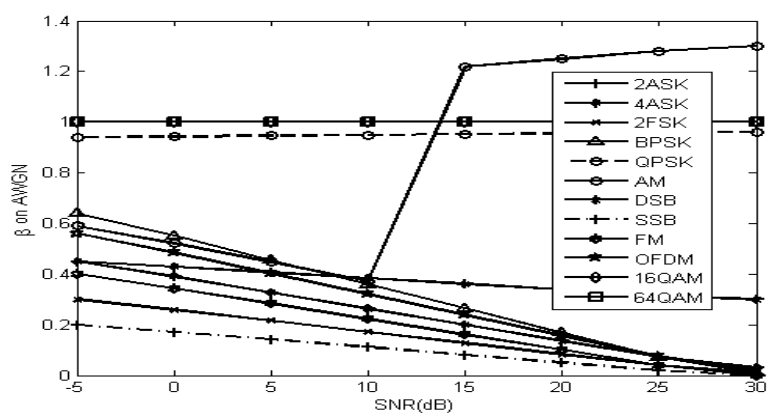

(e)

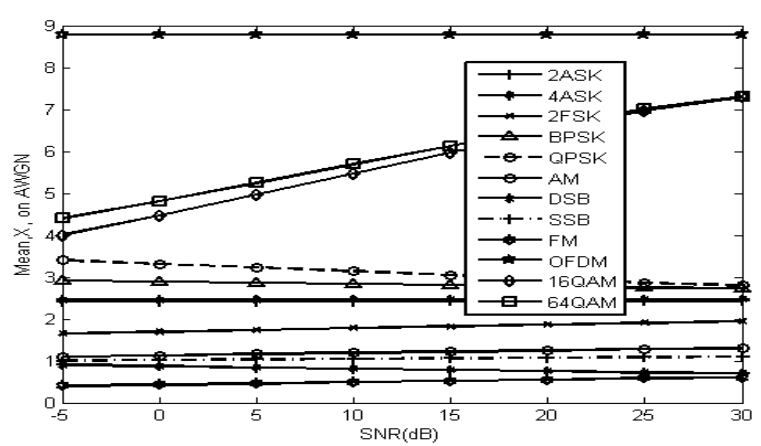

(f) 


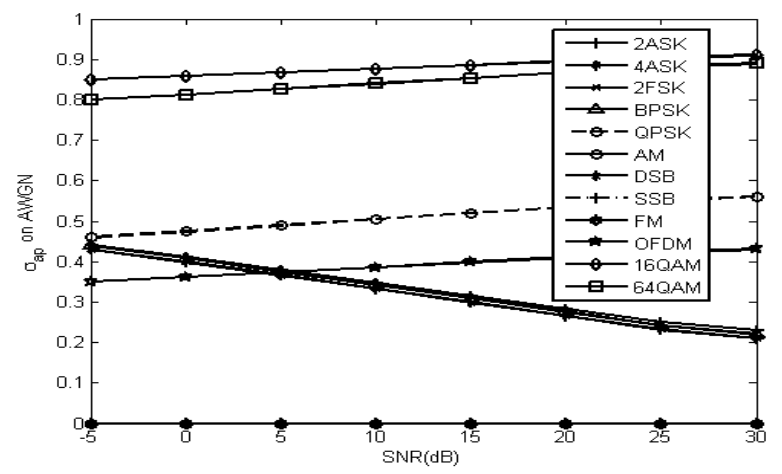

(g)

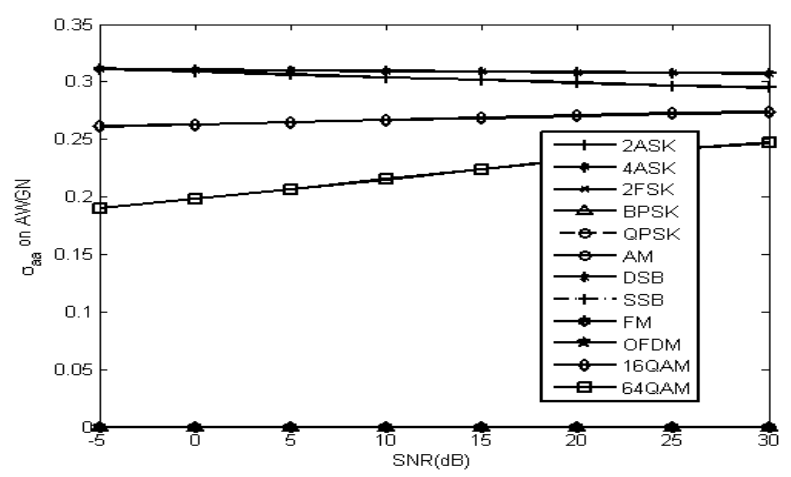

(h)

Fig. 1. Variation of (a) $\gamma_{\max }$, (b) $\sigma_{d p}$, (c) $\mathrm{P}$, (d) $v_{20}$, (e) $\beta$, (f) Mean, X, (g) $\sigma_{a p}$ and (h) $\sigma_{a a}$ with SNR for combined analog and digital modulated signals

\subsection{AMR pattern recognition approach second subsystem}

The activities in this subsystem are basically on the actual development and training of the proposed classifiers, which process those extracted feature keys and determine the modulation type of the received signal according to a predesigned decision rule. The classifiers were developed using an ANN, which is defined in [32] as an abstract representation of the biological nervous system. It consists of a collection of neurons that communicate with each other through axons. It is basically an adaptive system, which the interesting attributes it exhibits makes it capable of learning, adapting and generalizing. Artificial neural networks (ANNs) are classified according to either their architecture or the learning (training) algorithm.

According to their architecture, ANNs can be classified as either feed forward networks or recurrent networks. When ANNs are classified based on the learning or training algorithm employed, it can be supervised learning, unsupervised learning or reinforcement learning. The ANN architecture that was used for this study is a feed-forward backpropagation network, which is also known as multilayer perceptron (MLP) while the training method used is the supervised learning method. The architecture of the developed classifier is shown in Fig. 2 having the statistical feature extracted keys plotted in Fig. 1(a)-(h) as the inputs. The MLP consists of one input layer, one hidden layer of computational nodes or neurons, and one output layer of computational neurons. All the neurons are fully connected, as shown in Fig. 2. Eight neurons were used at the input layer corresponding to the number of input features, and fifteen neurons were used at the hidden layer. The network has twelve neurons at the output layer corresponding to the number of targets; that is the twelve combined analog and digital modulation schemes. The specifications for the developed AMR classifier are shown in Table 1.

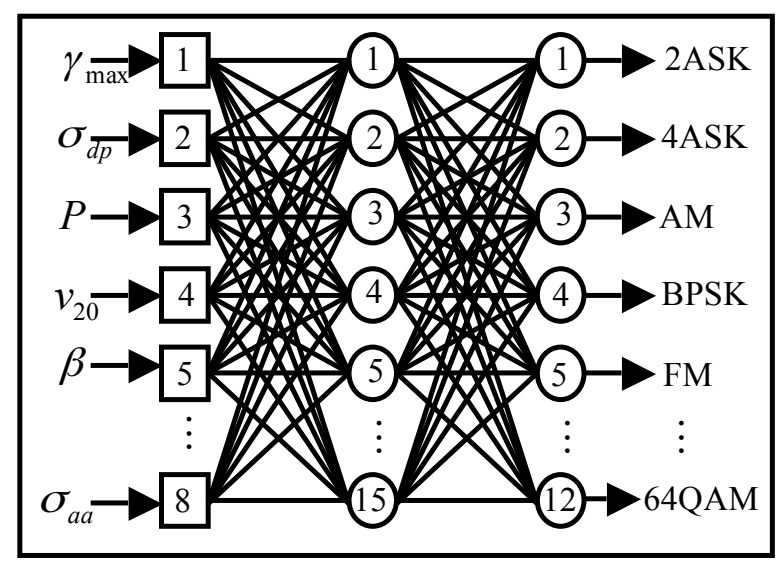

Fig. 2: Multilayer Feed-Forward Network Architecture for the developed AMR

Table 1. Specifications for the developed AMR

\begin{tabular}{|c|c|c|}
\hline Item & Parameters & Value \\
\hline 1. & $\begin{array}{l}\text { Type of neural network } \\
\text { architecture }\end{array}$ & Feed-forward \\
\hline 2. & No. of neurons in input layer & 8 \\
\hline 3. & $\begin{array}{l}\text { No. of neurons in hidden } \\
\text { layer }\end{array}$ & 15 \\
\hline 4. & $\begin{array}{l}\text { No. of neurons in output } \\
\text { layer }\end{array}$ & 12 \\
\hline 5. & Coefficient of weight-decay & 0.01 \\
\hline 6. & $\begin{array}{l}\text { Activation function } \\
\text { hidden layer }\end{array}$ & $\tanh$ \\
\hline 7. & $\begin{array}{l}\text { Activation function in output } \\
\text { layer }\end{array}$ & logistic \\
\hline 8. & Maximum number of epochs & 150 \\
\hline 9. & Performance function & $\begin{array}{l}\text { Mean Square Error } \\
\text { (MSE) }\end{array}$ \\
\hline 10. & Learning algorithm & $\begin{array}{l}\text { SCG and } \\
\text { CONJGRAD }\end{array}$ \\
\hline
\end{tabular}

During the training or learning process, input vectors and corresponding target vectors are used to train the network until it can classify the modulation schemes in an appropriate way. A total of 6000 data sets, with eight inputs and twelve target output, were used in developing the AMR classifiers for this study. The procedures followed to train the developed AMR classifiers are highlighted as follows:

The 6000 total generated data, consisting of inputs and target vectors, were imported into a MATLAB environment from an Excel spreadsheet. The loaded data were normalized and randomly sorted. The loaded data were partitioned into training, validation and testing data sets. Fifty percent of the generated data were used for the network training. The training data set was used to update the weights of the network. The network training was done until the MSE, which was used as the performance function, was minimal. Twenty-five percent of the total data were used to validate that the network was able to generalize. The last twenty-five percent of the total data were used as a completely independent test data to test the network generalization.

Finally, the AMR classifiers were developed. A feedforward network with non-linearity activation functions of tan-sigmoid (tanh) and logistic (log-sigmoid) were used in 
the hidden and output layers respectively in order to introduce non-linearity into the network because without non-linearity, the network will not be more powerful than plain perceptrons. The MLP was trained using SCG and CONJGRAD training algorithms. The two training algorithms were chosen not only to fulfil the main objective of this study but also because of their observed advantages in pattern recognition using ANNs. For instance, SCG was chosen because of its modest memory requirements with high accuracy and speed due to inexpensive calculation of the gradient information [33]. Similarly, the CONJGRAD training algorithm was chosen because it is also known to be a fast training algorithm with numerical efficiency and very low memory requirement [34].

Table 2. Developed AMR success recognition rate when trained with SCG

\begin{tabular}{ccccccc}
\hline \multirow{2}{*}{ Modulation scheme } & \multicolumn{5}{c}{$\begin{array}{c}\text { Performance of success recognition rate at different SNR value using 15 hidden neurons and 150 } \\
\text { training cycles on additive white Gaussian noise (AWGN) channel }\end{array}$} \\
\cline { 2 - 7 } & $-5 \mathrm{~dB}$ & $0 \mathrm{~dB}$ & $5 \mathrm{~dB}$ & $10 \mathrm{~dB}$ & $15 \mathrm{~dB}$ & $20 \mathrm{~dB}$ \\
\hline 2ASK & 97.55 & 99.46 & 99.66 & 99.84 & 99.91 & 99.97 \\
4ASK & 96.79 & 97.77 & 98.68 & 99.47 & 99.94 & 99.98 \\
2FSK & 99.22 & 99.65 & 99.79 & 99.84 & 99.97 & 99.99 \\
BPSK & 99.85 & 99.89 & 99.93 & 99.97 & 99.98 & 99.99 \\
QPSK & 99.54 & 99.64 & 99.88 & 99.92 & 99.97 & 99.98 \\
AM & 99.91 & 99.93 & 99.94 & 99.96 & 99.98 & 100.00 \\
DSB & 99.84 & 99.87 & 99.90 & 99.95 & 99.97 & 99.98 \\
SSB & 99.91 & 99.95 & 99.97 & 99.98 & 99.99 & 99.99 \\
FM & 99.93 & 99.95 & 99.96 & 99.97 & 99.99 & 99.99 \\
OFDM & 99.81 & 99.89 & 99.94 & 99.96 & 99.97 & 99.98 \\
16QAM & 98.89 & 99.15 & 99.88 & 99.91 & 99.95 & 99.99 \\
64QAM & 98.75 & 98.97 & 99.75 & 99.89 & 99.93 & 99.97 \\
\hline Overall success rate (\%) & $\mathbf{9 9 . 1 7}$ & $\mathbf{9 9 . 5 1}$ & $\mathbf{9 9 . 7 7}$ & $\mathbf{9 9 . 8 9}$ & $\mathbf{9 9 . 9 6}$ & $\mathbf{9 9 . 9 8}$ \\
\hline
\end{tabular}

Table 3. Developed AMR success recognition rate when trained with CONJGRAD

\begin{tabular}{|c|c|c|c|c|c|c|}
\hline \multirow[t]{2}{*}{$\begin{array}{l}\text { Modulation } \\
\text { scheme }\end{array}$} & \multicolumn{6}{|c|}{$\begin{array}{l}\text { Performance of success recognition rate at different SNR value using } 15 \text { hidden neurons and } 150 \\
\text { training cycles on additive white Gaussian noise (AWGN) channel }\end{array}$} \\
\hline & $-5 \mathrm{~dB}$ & $0 \mathrm{~dB}$ & $5 \mathrm{~dB}$ & $10 \mathrm{~dB}$ & $15 \mathrm{~dB}$ & $20 \mathrm{~dB}$ \\
\hline $2 \mathrm{ASK}$ & 87.10 & 99.91 & 99.94 & 99.97 & 99.99 & 99.99 \\
\hline 4ASK & 82.21 & 99.87 & 99.91 & 99.96 & 99.98 & 99.99 \\
\hline 2FSK & 99.56 & 99.88 & 99.93 & 99.95 & 99.98 & 99.99 \\
\hline BPSK & 99.92 & 99.95 & 99.96 & 99.97 & 99.99 & 100.00 \\
\hline QPSK & 99.42 & 99.76 & 99.84 & 99.96 & 99.98 & 99.99 \\
\hline $\mathrm{AM}$ & 99.57 & 99.89 & 99.94 & 99.97 & 99.98 & 99.99 \\
\hline DSB & 97.87 & 99.82 & 99.93 & 99.95 & 99.97 & 99.98 \\
\hline SSB & 99.90 & 99.94 & 99.95 & 99.97 & 99.99 & 100.00 \\
\hline FM & 99.93 & 99.95 & 99.97 & 99.98 & 99.99 & 99.99 \\
\hline OFDM & 99.48 & 99.91 & 99.94 & 99.96 & 99.98 & 99.98 \\
\hline 16QAM & 98.46 & 99.87 & 99.84 & 99.92 & 99.97 & 99.98 \\
\hline 64QAM & 93.66 & 98.91 & 99.65 & 99.87 & 99.94 & 99.99 \\
\hline $\begin{array}{l}\text { Overall success } \\
\quad \text { rate }(\%)\end{array}$ & 96.42 & 99.81 & 99.90 & 99.95 & 99.98 & 99.99 \\
\hline \multicolumn{7}{|c|}{ Average operational time $=\mathbf{1 0 . 6 4} \mathrm{ms}$} \\
\hline
\end{tabular}

\section{Results and Discussions}

This section is divided into two subsections. In the first subsection, the effects of the two training algorithms used on the performance of the propose AMR classifiers are presented and analysed. In the second subsection, the performance evaluation of the propose AMR classifiers are further assessed by comparing their overall success recognition rates using the two training algorithms employed with another study on joint analog and digital AMR classifier. The results of the two comparative studies are presented and discussed using tables and figures

\subsection{Effect of training algorithms on the propose AMR classifiers}

After the development and training of the developed AMR classifiers, their performances were evaluated using $25 \%$ of the total generated data as a test data set. The performance evaluations were investigated on different SNR values of -5 , $0,5,10,15$ and $20 \mathrm{~dB}$, using the SCG and the CONJGRAD training algorithms. The success recognition rates and the average operational time taken when the AMR classifiers were run for 150 cycles, using the two training algorithms, SCG and CONJGRAD, with the same test input data sets are presented in Table 2 and Table 3 respectively. 
The results of the performance evaluation of the developed AMR classifiers with the two training algorithms show, that the AMR classifiers could correctly and accurately classify the twelve combined analog and digital modulation schemes considered with an average success rate above $99.0 \%$ for all the simulated signals with SNR values from $0 \mathrm{~dB}$ upward without a priori knowledge of the signals parameters. However, for signal at $-5 \mathrm{~dB} \mathrm{SNR}$, the performances varied slightly and where the SCG classifier outperformed that of CONJGRAD. In addition, with the two training algorithms employed, the results obtained show a progressive increase in the success recognition rates as the SNR value increases. However, apart from the observed variation in success recognition rates of these two training algorithms when the same sets of testing data are used, one significant difference between the two classifiers developed with these two training algorithms is in their operational time taken. The comparative results on operational time taken show that SCG classifier acts faster than CONJGRAD classifier with average operational time taken of about 4.07 milliseconds, while that of CONJGRAD classifier is above 10.00 milliseconds. These variations in recognition values as well as the significant difference in their average operational time taken for the two classifiers under the same conditions, confirms that different training algorithms contribute significantly to ANN classifier performance.

A further comparative analysis was also performed in order to measure the performance of the two training algorithms quantitatively. This was done by plotting the operating characteristic curves for the two training algorithms as shown in Fig. 3. From this graph, it is observed that apart from the SNR value $-5 \mathrm{~dB}$ where SCG classifier outperforms CONJGRAD classifier, the CONJGRAD classifier outperforms SCG classifier in all the other five SNR values considered. The graph also revealed that apart from the faster response rate observed with the classifier developed using SCG training algorithm, its successive recognition rate is slow compared with that of the CONJGRAD classifier as the SNR values increase. The results of this study, therefore, established the fact that ANN classifier developed by SCG training algorithm responded faster than the corresponding one developed by CONJGRAD training algorithm. However, the classifier developed by CONJGRAD training algorithm exhibits better classification efficiency than the classifier developed using SCG training algorithm. Hence, in an application where speed is the priority, SCG training algorithm will be preferred. On the other hand, if classification efficiency is the priority, it is advisable to employ CONJGRAD training algorithm.

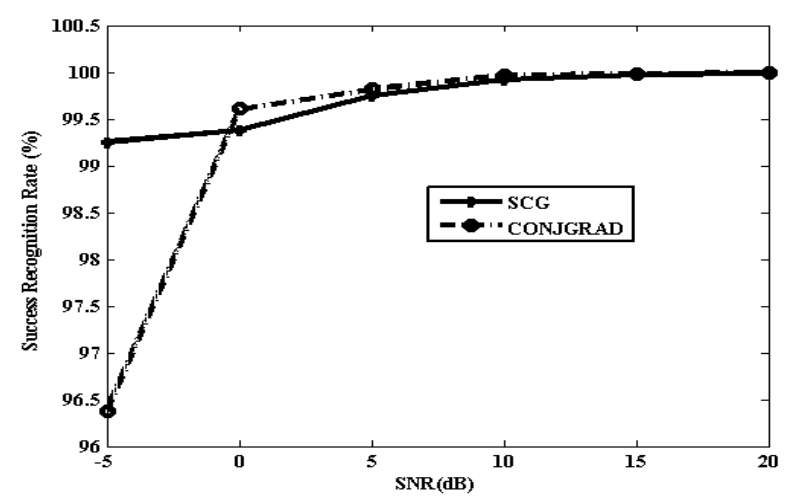

Fig. 3. SCG and CONJGRAD operating characteristic curves

\subsection{Comparison with previous study}

In order to evaluate the performance of the two AMR classifiers developed in this study, their overall success recognition results were compared with the results obtained from a similar classifier for automatic recognition of joint analog and digital modulation schemes. Specifically, the reference classifier used is characterized by (i) equal value of SNR; (ii) same set of modulation schemes, and (iii) absence of any a priori knowledge assumption on the signal characteristics. However, the differences between this study and the reference study reported in [35] are differences in feature keys employed and approach employed in developing the classifier. While our study uses eight distinct feature keys to discriminate between twelve combined analog and digital modulation schemes using PR approach, the classifier developed in [35] used three distinct feature keys to discriminate between nine joint analog and digital modulation schemes using the DT approach.

Apart from the similarities between the classifiers developed in this study and that developed in [35] that enhance the possibility of comparing the classifiers, the choice of [35] was made because it provides an opportunity to compare the results obtained from classifier developed using the PR approach with the DT approach. The latter is currently acclaimed as the approach that gives the most optimal result. The comparative overall success recognition rates of the classifiers proposed in this study with the two training algorithms and classifier developed in [35] are presented in Fig. 4. Excluding 2FSK and QPSK that were $100 \%$ recognized in [35] and over $99.80 \%$ recognized in this study, all other similar modulation schemes (AM, FM and BPSK) considered were recognized with higher success recognition rates than results presented in [35]. This shows that the two classifiers in this study perform favourably with previous classifiers surveyed literature. The result of the comparative overall success recognition rates also shows that the procedures involved in developing the two classifiers in this study are accurate. Furthermore, the result of the overall success recognition rates in the comparative study supports the observation made by [4] that if the PR approaches are carefully designed, they can achieve nearly optimal performance.

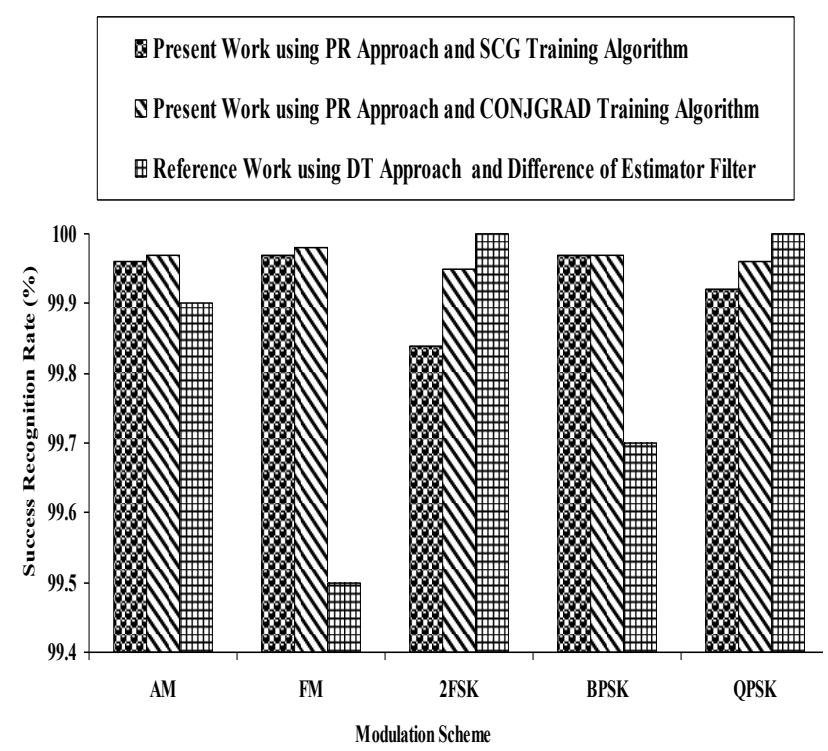

Fig. 4. Success recognition rate comparison between the present work and reference work in [35] at equal SNR $=10 \mathrm{~dB}$ 


\section{Conclusion}

This paper presents the overall procedural stages involved in developing and evaluating joint automatic analog and digital modulation classifiers that classify combined analog and digital communication signals without any a priori knowledge of the modulation schemes of the signals using a PR approach. The classifier evaluation is done using extensive simulations of twelve combined analog and digital modulated signals. The sample results of SNRs introduced at $-5,0,5,10,15$ and $20 \mathrm{~dB}$ for the considered combined analog and digital modulated signals without any preknowledge of the modulation schemes and modulation parameters, show that the developed classifiers are accurate and reliable. The sample results for the two classifiers developed with the two training algorithms employed show good success recognition performance with an average overall recognition rate above $99.50 \%$ at SNR value from 0 $\mathrm{dB}$ and above, and an average overall recognition rate slightly above $99.00 \%$ and $96.40 \%$ respectively at $-5 \mathrm{~dB}$ SNR value for SCG trained classifier and CONJGRAD trained classifier.

Likewise, the comparative investigation carried on the effect of the training algorithms on the performance of the developed ANN classifiers in this study shows that different training algorithms have different impacts on an ANN classifier. The comparative results show that while the classifier developed using the SCG training algorithm shows faster response rate than the classifier developed using the
CONJGRAD training algorithm, the CONJGRAD trained classifier shows better progressive recognition efficiency than that developed using the SCG training algorithm. Furthermore, the comparison assessment carried out to evaluate the performance of the classifiers developed in this study using the PR approach, and the previous study that uses the DT approach, shows that the developed classifiers for this study perform favourably with the study that uses the DT approach. The performance of the two classifiers developed for this study therefore demonstrate that classifiers developed using a PR approach can compare favourably with classifiers developed using a DT approach when well designed.

\section{Acknowledgment}

The authours thank all the sponsors of the University of the Witwatersrand's Centre for Telecommunications Access and Services (CeTAS) for their financial support. The authors also express their appreciation to the Independent Communications of South Africa (ICASA) for its financial support. The principal author also acknowledges the financial assistance received from the University of the Witwatersrand's Postgraduate Merit Award (PMA).

\section{References}

1. L.V. Dominguez, J.M.P. Borrallo, and J.P. Garcia: “A general approach to the automatic classification of radiocommunication signals", Signal Processing, Vol. 22, No. 3, pp. 239-250, March 1991.

2. H. Guldemir and A. Sengur: "Online modulation recognition of analog communication signals using neural network", Expert System with Applications, Vol. 33, pp. 206-214, July 2007.

3. A.I.R. Frontes, L.C.P. Cavalcante, and L.F.Q Silveira: "Novel automatic modulation classification using correntropy coefficient", Proceedings of Brazilian Symposium on Telecommunications 2012 (SBrT 2012), 13-16 September 2012, Brasilia DF, pp. 1-5. Available [Online]: http://sbrt.org.br/sbrt2012/publicacoes/98843 1.pdf. Access on 1st August, 2013.

4. H.C. Wu, M. Saquib and Z. Yun: "Novel Automatic Modulation Classification Using Cumulant Features for Communications via multipath Channels", IEEE Transaction on Wireless Communications, Vol. 7 No. 8, pp. 3098-3105, August 2008.

5. Y.T. Chen, L.G. Gadbois, and P. Yansouni: "Identification of the modulation type of a signal", Proceedings of IEEE International Conference on Acoustics, Speech, and Signal Processing 1985 (ICASSP' 85), Vol. 10, pp. 838-841, April 1985.

6. P.M. Fabrizi, L.B. Lopez, and G.B. Lockhart: "Receiver recognition of analogue modulated types," Proceedings of IERE Conference on Radio Receiver and associated Systems. Bangor, Wales, pp. 135-140, 1986.

7. Y.O. Al-Jalili: "Identification algorithm of upper sideband and lower sideband SSB signals", Signal Processing, Vol. 42, No. 2, pp. 207-213, March 1995.

8. E.E. Azzouz, and A.K. Nandi: "Automatic Modulation Recognition - 1", Journal of Franklin Institute, Vol. 334B, No. 2, pp. 241-273, March 1997.

9. A. Sengur, and H. Guldemir: "An educational interface for automatic recognition of analog modulated signals", Journal of Applied Sciences, Vol. 5, No. 3, pp. 513-516, 2005.

10. F.F. Liedtke: "Computer simulation of an automatic classification procedure for digitally modulated communication signals with unknown parameters", Signal Processing, Vol. 6, Nol. 4, pp. 311323, August 1984.

11. M.P. DeSimio and G.E. Prescott: "Adaptive generation of decision functions for classification of digitally modulated signals", Proceedings of IEEE National Aerospace and Electronics Conference 1988 (NAECON 1988), Dayton OH, US, pp. 1010-1014, 23 - 27 May 1988.

12. E.E. Azzouz and A.K. Nandi: "Automatic identification of digital modulations", Signal Processing, Vol. 47, No. 1, pp. 55-69, November 1995.

13. S. Taira: "Automatic classification of QAM signals by neural networks", Proceedings of IEEE International Conference on Acoustics, Speech, and Signal Processing 2001 (ICASSP, 2001), Salt Lake City UT, US, Vol. 2, pp. 1309-1312, 7 - 11 May 2001.

14. M.L.D. Wong, and A.K. Nandi: "Automatic digital modulation recognition using artificial neural network and genetic algorithm", Signal Processing, Vol. 84, No. 2, pp. 351-365, February 2004.

15. A. Kubankova, and D. Kubanek: "Extended method of digital modulation recognition and its testing", RadioEngineering, Vol. 20, No. 1, pp. 25-30, April 2011.

16. F. Jondral: "Automatic classification of high frequency signals", Signal Processing, Vol. 9, No. 3, pp. 177-190, October 1985.

17. A.K. Nandi and E.E. Azzouz: "Algorithms for automatic modulation recognition of communications signals", IEEE Transactions on Communications, Vol. 46, No. 4, pp. 431-436, April 1998.

18. O.A. Dobre, A. Abdi, Y. Bar-Ness and W. Su: "The classification of joint analog and digital modulations", Proceedings of IEEE Military Communications Conference 2005 (IEEE MILCOM 2005), Atlantic City, NJ, Vol. 5, pp. 3010-3015, 17 - 20 October, 2005 .

19. O.A. Dobre, A. Abdi, Y. Bar-Ness, and W. Su: "Survey of automatic modulation classification techniques: classical approaches and new trends", IET Communications, Vol. 1, No. 2, pp. 137-156, April 2007. 
20. A.E. Zadeh, S.A. Seyedin, and M. Dehghan: "An intelligent method for modulation type identification", Proceedings of $3 \mathrm{rd}$ International Conference on Mobile Technology, Applications and Systems, Bangkok, Thailand, pp. 1-4, 25 - 27 October 2006.

21. W. Wei and J.M. Mendel: "A fuzzy logic method for modulation classification in non idea environments", IEEE Transactions on Fuzzy Systems, Vol. 7, pp. 333-344, June 1999.

22. J. Lopatka, and M. Pedzisz: "Automatic modulation classification using statistical moments and fuzzy classifier", Proceedings of IEEE 5th International Conference on Signal Processing 2000 (IEEE-ICSP 2000), Beijing, China, Vol. 3, pp. 1500-1506, August 2000.

23. B.G. Mobasseri: "Constellation shape as a robust signature for digital modulation recognition", Proceedings of IEEE Military Communications Conference 1999 (MILCOM 1999), Atlantic City, NJ, pp. 442-446, 31 October - 3 November 1999.

24. M.L.D. Wong and A.K. Nandi: "Automatic digital modulation recognition using spectral and statistical features with multi-layer perceptrons", Proceedings of the 6th International Symposium on Signal Processing and its Applications 2001 (ISSPA 2001),Kuala Lumpur, Malaysia, pp. 390-393, 13 - 16 August 2001.

25. J.J. Popoola, and R. van Olst: "Automatic recognition of analog modulated signals using artificial neural networks", Journal of Computer Technology and Applications, Vol. 2, No. 1, pp. 29-35, January 2011.

26. E.E. Azzouz and A.K. Nandi: "Automatic Modulation Recognition of Communication Signals", Kluwer Academic Publishers, Boston, pp. 46-47 \& pp. 84-86, September1996.

27. O. Mahmoud, F. Anwar, and M.J.E. Salami: "Learning algorithm effect on multilayer feed forward artificial neural network performance in image coding", Journal of Engineering Science and Technology, Vol. 2, No. 2, pp. 188-199, August 2007.

28. G.P. Zhang: "Neural networks for classification: A survey", IEEE Transactions on Systems, Man, and Cybernetics-Part C:
Applications and Reviews, Vol. 30, No. 4, pp. 451-462, November 2000.

29. N. An, B. Li, and M. Huang: "Modulation classification of higher order MQAM signals using mixed-order moments and Fisher criterion", Proceedings of the 2nd IEEE International Conference on Computer and Automation Engineering 2010 (ICCCA 2010), Singapore, pp. 150-153, 26 - 28 February 2010.

30. B. Wang and L. Ge: "A novel algorithm for identification of OFDM signal", Proceedings of International Conference on Wireless Communications, Networking and Mobile Computing 2005 (WCNM 2005), pp. 261- 264, 2005, 23 - 26 September 2005.

31. F.-Q. Huang, Z.-M., Zhong, Y.-T. Xu, and G.C. Ren: "Modulation recognition of symbol shaped digital signals", Proceedings of International Conference on Communications, Circuits and Systems 2008 (ICCCAS 2008), Fujian, pp. 328-332, $25-27$ May 2008.

32. J.P.T. Yusiong: "Optimizing artificial neural networks using cat swarm optimization algorithm", International Journal of Intelligent Systems and Applications, Vol. 5, No. 1, pp. 69-80, Vol. 1, December 2012.

33. N. Mohamad, F. Zaini, A. Johari, I. Yassin, and A. Zabidi: "Comparison between Levenberg-Marquardt and Scaled conjugate gradient training algorithms for breast cancer diagnosis using MLP", Proceedings of 6th International Colloquium on Signal Processing and It Applications 2010 (CSPA 2010), Mallaca City, Malaysia, pp. 1-7, 21 - 23 May, 2010.

34. D. Shanthi, G. Sahoo, and N. Saravanan: "Comparison of neural network training algorithms for the prediction of the patient's post-operation recovery area", Journal of Convergence Information Technology, Vol. 4, No. 1, pp. 24-32, March 2009.

35. M. Kang, C. Lee, and J. Joo: "Automatic recognition of analog and digital modulation signals using DoE filter", Proceedings of the 9th International Symposium on Communications and Information Technologies 2009 (ISCIT 2009), pp. 609-614, 28 30 September 2009. 